\title{
Low podoplanin expression in pretreatment biopsy material predicts poor prognosis in advanced-stage squamous cell carcinoma of the uterine cervix treated by primary radiation
}

\author{
Kimberly L Dumoff ${ }^{1}$, Christina S Chu², Eleanor E Harris ${ }^{3}$, David Holtz ${ }^{4}$, Xiaowei Xu ${ }^{1}$, \\ Paul J Zhang ${ }^{1}$ and Geza Acs ${ }^{1}$ \\ ${ }^{1}$ Department of Pathology and Laboratory Medicine, University of Pennsylvania Medical Center, \\ Philadelphia, PA, USA; ${ }^{2}$ Division of Gynecologic Oncology, Department of Obstetrics and Gynecology, \\ University of Pennsylvania Medical Center, Philadelphia, PA, USA; ${ }^{3}$ Department of Radiation Oncolology, \\ University of Pennsylvania Medical Center, Philadelphia, PA, USA and ${ }^{4}$ Department of Obstetrics and \\ Gynecology, Lankenau Hospital, Wynnewood, PA, USA
}

\begin{abstract}
Lymphatic invasion and nodal metastasis are predictors of poor outcome in cervix carcinoma. We have recently found that low podoplanin immunoreactivity in cervix carcinoma correlated with the presence of lymphatic invasion and nodal metastasis. In the current study, we examined whether podoplanin expression in pretreatment cervical biopsies can predict the presence lymphatic invasion, nodal metastasis, and outcome in advanced-stage tumors treated by nonsurgical means. Podoplanin expression was analyzed by immunohistochemistry in $\mathbf{4 8}$ cervical biopsies and corresponding hysterectomy specimens of early-stage invasive squamous cell carcinoma and in $\mathbf{7 4}$ pretreatment biopsies from advanced-stage tumors treated with primary radiation. We found a highly significant correlation between podoplanin expression obtained in biopsy and corresponding hysterectomy materials $(r=0.8962, P<0.0001)$. Low podoplanin expression showed a significant correlation with lymphatic invasion $(P<0.0001)$ and nodal metastasis $(P=0.0058)$. Low podoplanin expression in pretreatment biopsy material showed a significant correlation with poor disease-free $(P=0.0009)$ and overall $(P=0.0002)$ survival in advanced-stage tumors. Our results suggest that in advanced-stage cervix carcinomas treated by radiation, when traditional prognostic indicators are not available and treatment decisions are based on biopsy material and clinical staging parameters, examination of podoplanin expression in pretreatment biopsy material may be a useful marker to predict lymphatic metastasis and patient outcome. Prospective studies involving larger numbers of patients are needed to further evaluate the clinical utility of examination of podoplanin expression in patients with cervix carcinoma.
\end{abstract}

Modern Pathology (2006) 19, 708-716. doi:10.1038/modpathol.3800580; published online 10 March 2006

Keywords: podoplanin; D2-40 antibody; cervix carcinoma; lymphatic invasion; lymph node metastasis; prognostic marker

In most solid tumors, including cervical cancer, the spread of cancer cells via the lymphatics to regional lymph nodes is an important early event in metastatic disease. ${ }^{1,2}$ Nodal metastasis is a key factor in the staging of human cancers, an important prog-

Correspondence: Dr G Acs, MD, PhD, Department of Interdisciplinary Oncology, H Lee Moffitt Cancer Center and Research Institute, Room 2071M, 12902 Magnolia Drive, Tampa, FL 33612, USA.

E-mail: acsg@moffitt.usf.edu

Presented in part at the 94th Annual Meeting of the United States and Canadian Academy of Pathology, February 26-March 4, 2005, San Antonio, TX, USA.

Received 27 December 2005; revised and accepted 07 February 2006; published online 10 March 2006 nostic indicator, and necessitates the treatment of regional lymph nodes by surgery and radiation. ${ }^{3}$ Indeed, lymphatic invasion and nodal metastasis have been found to be predictors of shorter diseasefree and overall survival in carcinoma of the uterine cervix. ${ }^{4-6}$ Patients with locally advanced disease (stages IIB-IV), however, are treated primarily with radiation and radiosensitizing chemotherapy to the primary tumor and the pelvic lymph nodes after biopsy confirmation of the diagnosis. Although this approach can control disease in the pelvis, microscopic spread to areas outside of the pelvis, such as the para-aortic nodes, are not included in pelvic radiation fields. Thus, extended-field radiation is typically administered to patients with documented 
para-aortic node involvement. While radiographic staging of para-aortic nodes is considered acceptable, although with a lower sensitivity and specificity, such documentation often requires separate surgical staging, typically via extraperitoneal or laparoscopic lymph node dissection. Aside from extra cost and patient morbidity, some investigators have reported significantly worse disease-free and overall survival among patients surgically staged compared to those clinically staged. ${ }^{7}$ Extended-field radiation itself incurs a high rate of intestinal morbidity $^{8,9}$ and prophylactic treatment of the para-aortic region has not been conclusively shown to provide any survival benefit. ${ }^{10,11}$ Unfortunately, in patients with unresectable disease, cervical biopsy material alone does not provide reliable histopathologic features to predict the presence of lymphatic invasion, nodal metastasis, or patient outcome to guide in therapy.

The recently developed monoclonal antibody D2-40 detects a fixation-resistant epitope on podoplanin. ${ }^{12,13}$ It was shown to be a selective marker for lymphatic endothelium allowing the specific identification of lymphatic vessels and the study of lymphatic vessel density in solid tumors. ${ }^{13-17} \mathrm{We}$ have recently found that cells of cervical squamous cell carcinomas also express podoplanin and characterized the D2-40 immunostaining pattern in a series of tumors treated by radical surgery. ${ }^{16}$ We found a highly significant correlation between the level of podoplanin expression and the presence of lymphatic invasion, nodal metastasis, and poor outcome in this cohort of patients with early-stage tumors. ${ }^{16}$ However, it was not clear whether podoplanin expression detected in small tumor tissue from cervical biopsies could be used to predict lymphatic invasion in the main tumor mass and nodal metastasis, which are usually better evaluated in hysterectomy specimens with lymph node staging biopsies. Similarly, it remained unclear whether examination of podoplanin expression in pretreatment biopsy materials obtained from advanced-stage tumors treated by nonsurgical means could be used as a prognostic and/or predictive marker in such cases.

The current study was designed to examine whether the level of podoplanin expression using D2-40 immunohistochemistry in limited cervical biopsy material is reflective of the immunoreactivity of the main tumor mass, and whether it can be a useful marker to predict the presence of nodal metastasis, lymphatic invasion, and clinical outcome in advanced-stage cases treated with nonsurgical methods.

\section{Materials and methods}

In order to determine whether podoplanin immunoreactivity in biopsy material corresponds to the expression pattern of the entire tumor mass and can predict the presence of lymphatic invasion and metastasis to regional lymph nodes, we identified 48 cases of invasive squamous cell carcinomas of the cervix (Series A) in the Surgical Pathology files of the University of Pennsylvania Medical Center. In all 48 cases adequate biopsy and corresponding hysterectomy specimens were available for the study. To determine whether podoplanin expression in pretreatment biopsy material can be used as a prognostic and/or predictive marker in advancedstage tumors treated by nonsurgical methods, 74 additional pretreatment biopsy samples of invasive squamous cell carcinoma treated by radiation (Series B) were selected. Hematoxylin and eosinstained slides of all cases were reviewed and the diagnoses confirmed. All available sections of the biopsy and hysterectomy materials were examined for the presence of lymphatic invasion. Staging was defined according to the International Federation of Obstetrics and Gynecology (FIGO) clinical staging system. ${ }^{18}$ Tumor grade was determined according to established criteria. ${ }^{19}$

The clinicopathologic features of the tumors included in both Series are summarized in Table 1. For Series A, pelvic/para-aortic lymph node dissection was performed in all cases; the mean and median number of lymph nodes per case examined was 25.9 and 24.0, respectively (range 6-82). Lymph node metastasis was present in $19(40 \%)$ cases; the mean and median number of positive lymph nodes was 2.5 and 2.0, respectively (range 1-7). Primary treatment was surgical in all cases; 23 patients received adjuvant treatment consisting of radiation in 16, and combination radiation and chemotherapy in seven cases, respectively.

For Series B, pelvic/para-aortic lymph node dissection was performed in $22(30 \%)$ cases; the mean and median number of lymph nodes per case examined was 8.0 and 7.0, respectively (range 1-26). Lymph node metastasis was present in 10 cases; the mean and median number of positive lymph nodes was 2.1 and 1.0, respectively (range 1-7). Patients were treated between May 1987 and May 2000. Of the 74 patients treated with definitive radiation, 70 received pelvic radiation, with or without paraaortic extended fields, to a median dose of $3960 \mathrm{cGy}$ (range 3060-6480 cGy). Implants were performed in 68 patients, as follows: tandem and ovoid intracavitary implant with Cesium-137 isotopes in 59 patients; Syed interstitial implants using Iridium192 isotopes in five patients; tandem and cylinder in two patients; a combination of a tandem and ovoid and a Syed interstitial or a tandem and cylinder in one patient each, respectively. One patient had brachytherapy alone to a point A dose of $6888 \mathrm{cGy}$. One patient had pelvic radiation alone to a dose of 6840 cGy. Data describing radiation treatment were not available for three patients. For patients treated with pelvic radiation followed by tandem and ovoid intracavitary implants, the combined median dose to the pelvis and point A average was $8111 \mathrm{cGy}$ 
Table 1 Summary of clinicopathologic features

\begin{tabular}{|c|c|c|}
\hline & $\begin{array}{c}\text { Series } A(\mathrm{n}=48), \text { cases } \\
\text { treated by radical } \\
\text { hysterectomy }\end{array}$ & $\begin{array}{l}\text { Series } B(\mathrm{n}=74) \text {, } \\
\text { cases treated by } \\
\text { radiation }\end{array}$ \\
\hline \multicolumn{3}{|l|}{ Age } \\
\hline Median & 45 & 52.5 \\
\hline Mean & 46.1 & 54.8 \\
\hline \multicolumn{3}{|l|}{ Tumor size $(\mathrm{cm})$} \\
\hline Median & 3.4 & 6.0 \\
\hline Mean & 3.5 & 5.6 \\
\hline \multicolumn{3}{|c|}{$\begin{array}{l}\text { Depth of invasion } \\
(\mathrm{mm})\end{array}$} \\
\hline Median & 10.0 & N/A \\
\hline Mean & 16.1 & N/A \\
\hline \multicolumn{3}{|c|}{ Lymphatic invasion } \\
\hline Absent & 15 & N/A \\
\hline Present & 33 & 16 \\
\hline Unknown $^{\mathrm{a}}$ & 0 & 56 \\
\hline \multicolumn{3}{|c|}{ Nodal metastasis } \\
\hline Absent & 29 & 12 \\
\hline Present & 19 & 10 \\
\hline Unknown $^{\mathrm{b}}$ & 0 & 52 \\
\hline \multicolumn{3}{|l|}{ FIGO stage } \\
\hline IA & 7 & 0 \\
\hline IB & $34^{\mathrm{c}}$ & 20 \\
\hline IIA & 7 & 2 \\
\hline IIB & 0 & 30 \\
\hline IIIA & 0 & 6 \\
\hline IIIB & 0 & 12 \\
\hline IVA & 0 & 2 \\
\hline IVB & 0 & 2 \\
\hline \multicolumn{3}{|l|}{ Tumor grade } \\
\hline Low & 3 & 0 \\
\hline Intermediate & 21 & 33 \\
\hline High & 24 & 41 \\
\hline
\end{tabular}

${ }^{\mathrm{a}}$ In Series B, where only biopsy samples were available for review, cases were considered positive for lymphatic invasion if tumor cells within lymphatic spaces were seen on the biopsy samples; otherwise, lymphatic invasion status was considered unknown in these cases.

${ }^{\mathrm{b}}$ In series B, pelvic and/or para-aortic lymph node dissection was performed in 22 cases.

${ }^{\mathrm{c}} \mathrm{Six}$ of these patients had microscopic invasion of parametrial tissue by tumor.

(range 7500-9500 cGy). For patients treated with pelvic radiation and a Syed interstitial implant, the median combined dose to the pelvis and prescription isodose was $7518 \mathrm{cGy}$ (range 7040-9020 cGy). The doses to the remaining four patients with other types of implants were also within this combined dose range (7495-9540 cGy). Concurrent radiosensitizing chemotherapy was given in 26 patients, consisting of bolus 5-fluorouracil and cisplatin in nine patients and weekly cisplatin in 17 patients. Two patients received sequential chemotherapy.

Follow-up of patients was performed on the basis of information reported in the clinical histories. We considered as uncensored records of patients who died of disease; we considered as censored records of all patients who were alive at follow-up or patients who died of a cause not related to the disease. Study protocols were approved by the University of Pennsylvania Institutional Review Board.

Immunohistochemical assays were performed on formalin-fixed paraffin-embedded sections. Sections ( $5 \mu \mathrm{m}$ thick) were cut and deparaffinized in xylene and rehydrated in graded alcohols. Slides were boiled in $1 \times$ EDTA buffer (LabVision, Fremont, CA, USA) for $20 \mathrm{~min}$. Endogenous peroxidase activity was blocked by $3 \%$ hydrogen peroxide in methanol for $20 \mathrm{~min}$. Slides were incubated with the D2-40 monoclonal antibody (1:25 dilution, Signet Laboratories, Dedham, MA, USA) for $1 \mathrm{~h}$ at room temperature. Immunohistochemical staining was performed on a DAKOCytomation Autostainer using the EnVision + HRP DAB system (DAKOCytomation, Carpinteria, CA, USA), according to the manufacturer's recommendations.

Podoplanin (D2-40) immunoreactivity in the tumor cells was evaluated semiquantitatively on a fourtiered scale. ${ }^{16}$ Membranous and/or cytoplasmic immunoreactivity was considered positive. Strong immunostaining was defined as reactivity similar in intensity to that seen in lymphatic vessels present in the tissue sections, which were used as internal positive controls. The percentage of weakly, moderately, and strongly staining cells was determined, and a staining score was calculated as follows: Score (out of maximum of 300 ) $=$ sum of $1 \times$ percentage of weak, $2 \times$ percentage of moderate, and $3 \times$ percentage of strong staining. The immunohistochemical scores were used to compare reactivity in biopsy and corresponding hysterectomy materials. For the purpose of further statistical analyses, tumors were considered to show high podoplanin immunoreactivity if the percentage of tumor cells showing immunostaining of any intensity was higher than the median value $(10 \%)$ for all tumors. This simplified scoring system reliably stratified patients into two distinct groups with only 11 of $122(9 \%)$ patients showing immunoreactivity near the cutoff value.

The correlation between the immunostaining scores obtained in biopsy and hysterectomy materials was analyzed using the Spearman test. High vs low immunostaining in carcinomas was compared using the Fisher's exact test. Survival curves were plotted using the method of Kaplan and Meier and compared using the log-rank test. A Cox proportional hazards model was used to assess the effect of tumor variables on survival. The end point was the overall survival from the day of treatment. Statistical significance was determined if the two-sided $P$-value of a test was less than 0.05. Computations were performed using the SYSTAT (Version 10.2, SYSTAT Software Inc., Richmond, CA, USA) software.

\section{Results}

The results of the podoplanin immunohistochemical assays are illustrated in Figure 1. A highly 
significant positive correlation was found between the podoplanin immunostaining scores and percent immunoreactivity values obtained in biopsy and hysterectomy materials $(r=0.8835, P<0.0001$ and $r=0.8962, P<0.0001$, respectively, Spearman test) (Figure 2a and b). Based on the biopsy and hysterectomy materials, $16(38 \%)$ and $18(43 \%)$ tumors were classified as showing high podoplanin
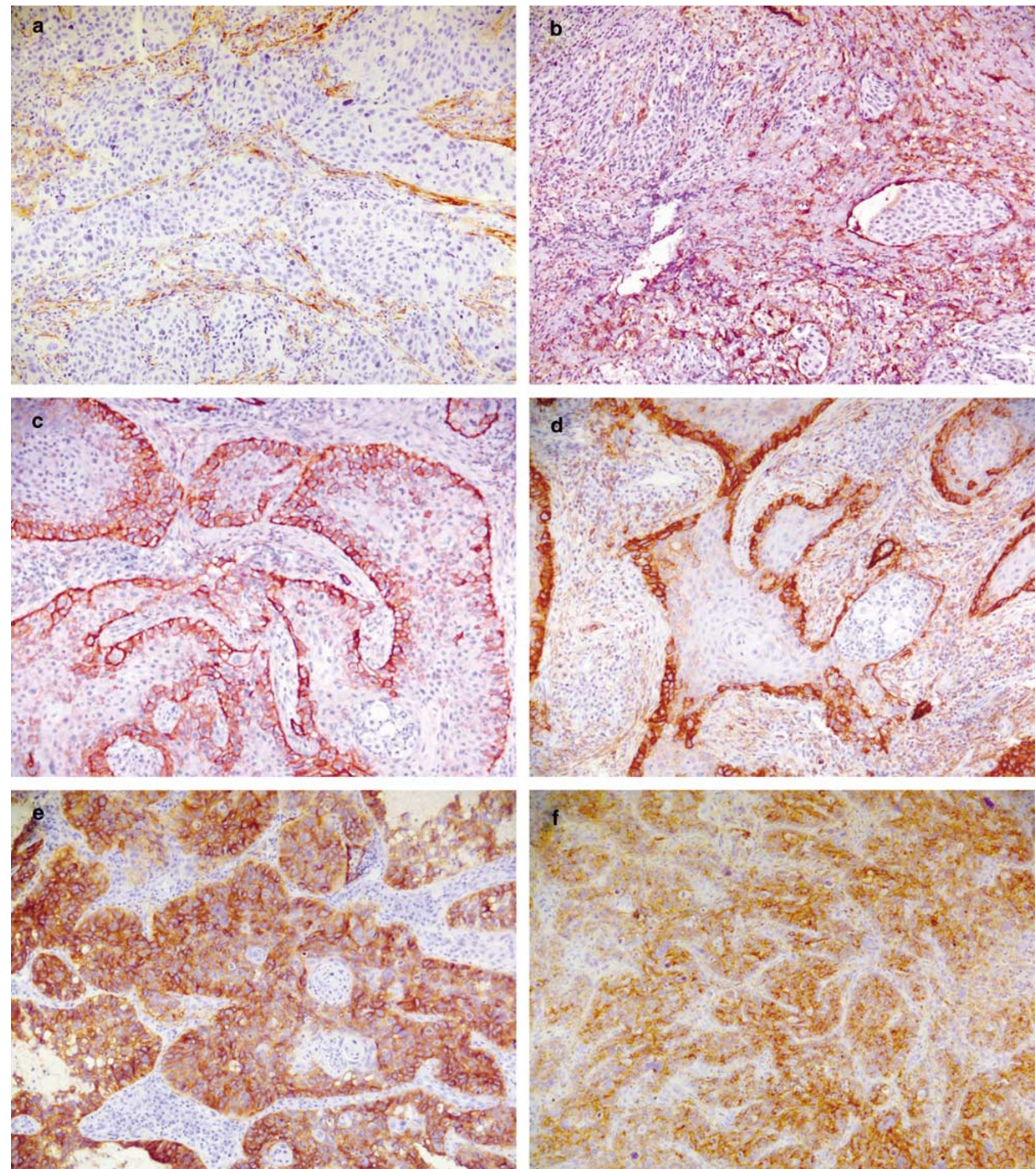

Figure 1 The pattern of podoplanin expression in squamous cell carcinoma of the cervix is very similar in biopsy samples (a, c, e) and hysterectomy specimens (b, d, f). (a and $\mathbf{b})$ The tumor cells show complete lack of podoplanin expression, while there is mild to moderate staining in the desmoplastic stroma. The D2-40 antibody strongly stains the lymphatic vessels and highlights the presence of lymphatic invasion. (c, d) Focal podoplanin expression in cervical squamous cell carcinoma is mainly localized to the periphery of invading tumor cell nests. (e, f) Strong, diffuse podoplanin expression in cervical squamous cell carcinoma. (Immunohistochemical stains for podoplanin with hematoxylin counterstain, original magnification $\times 100)$. 
expression, respectively. The difference between the results obtained in the two types of samples was statistically not significant $(P=0.8312$, Fisher's exact test).

Low podoplanin immunoreactivity in the tumors based on both biopsy and hysterectomy materials showed a highly significant association with the presence of lymphatic invasion $(P<0.0001$ both for biopsy and hysterectomy materials, Fisher's exact test $)$ and lymph node metastasis $(P=0.0058$ for biopsy and $P=0.0275$ for hysterectomy materials,
Fisher's exact test) (Figure 2c and d). The sensitivity, specificity, and positive and negative predictive values of low podoplanin expression in biopsy and hysterectomy materials to predict the presence of lymphatic invasion and nodal metastasis are summarized in Table 2.

Among the 74 advanced-stage cases treated by primary radiation (Series B), podoplanin expression was low in $39(53 \%)$ and high in $35(47 \%)$ cases, respectively. The frequency of low and high immunoreactivity was statistically not significantly
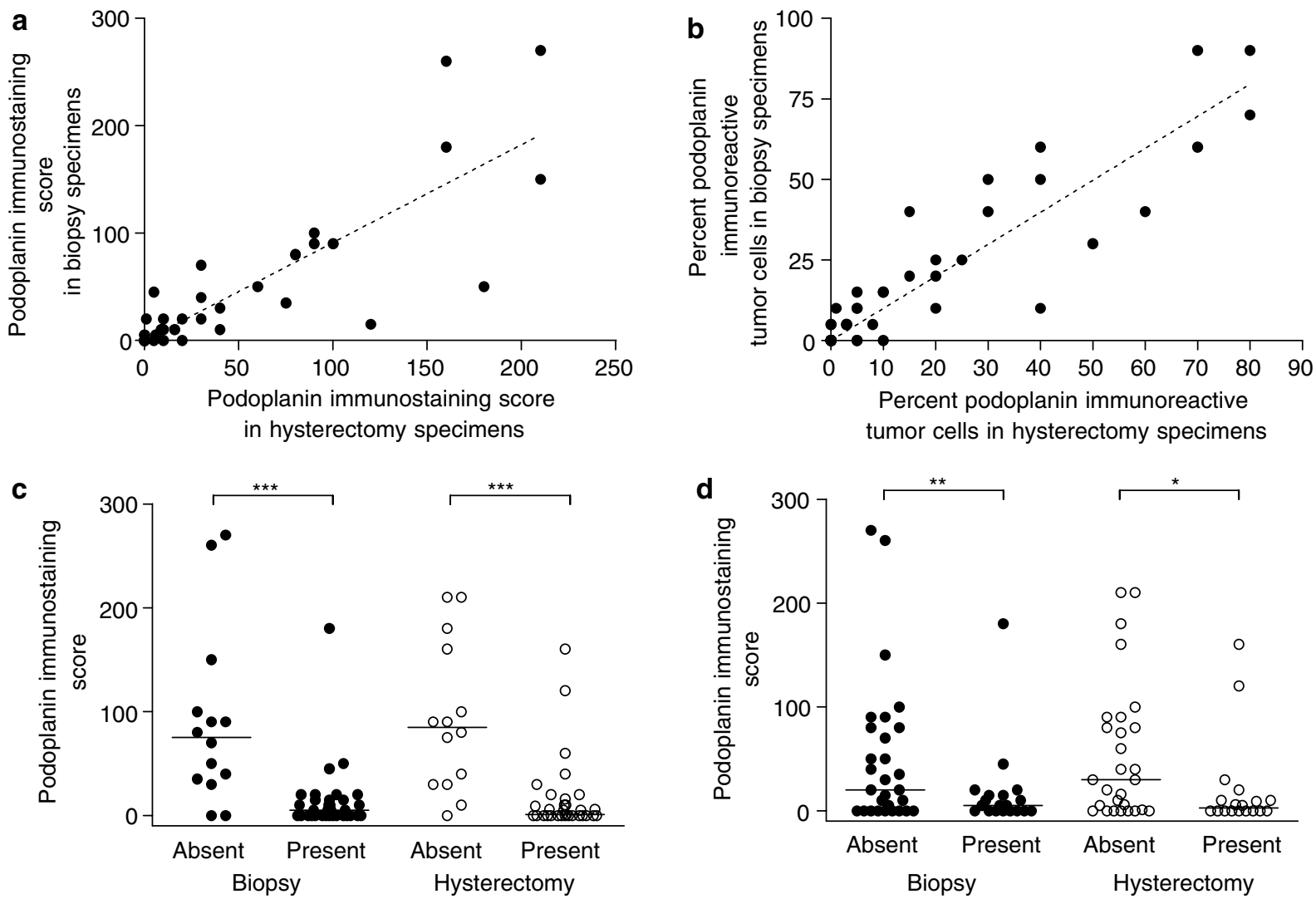

Figure 2 (a) Podoplanin immunostaining scores determined in hysterectomy and biopsy materials show a highly significant positive correlation $(r=0.8835, P<0.0001$, Spearman test). The dotted line represents the calculated regression line. (b) The percent of immunoreactive tumors cells determined in hysterectomy and biopsy materials show a highly significant positive correlation $(r=0.8962$, $P<0.0001$, Spearman test). The dotted line represents the calculated regression line. Podoplanin immunoreactivity is significantly lower in cases associated with the presence of lymphatic invasion (c) and nodal metastasis (d), based on both biopsy ( $(\bullet)$ and hysterectomy $(\bigcirc)$ material. Bars indicate the median values of podoplanin immunostaining scores $\left({ }^{*} P<0.05 ;{ }^{*} P<0.01\right.$; ${ }^{* * *} P<0.001$, Mann-Whitney test).

Table 2 Sensitivity, specificity, positive and negative predictive value of low podoplanin expression to predict the presence of lymphatic invasion and nodal metastasis in cervical squamous cell carcinomas

\begin{tabular}{|c|c|c|c|c|c|}
\hline & Sensitivity & Specificity & $P P V$ & $N P V$ & $R R(95 \% C I)$ \\
\hline \multicolumn{6}{|c|}{ Lymphatic invasion } \\
\hline Biopsy & 0.9091 & 0.8571 & 0.9375 & 0.8000 & $4.69(1.69-12.95)$ \\
\hline Hysterectomy & 0.8387 & 0.8571 & 0.9286 & 0.7059 & $3.16(1.50-6.64)$ \\
\hline \multicolumn{6}{|l|}{ Nodal metastasis } \\
\hline Biopsy & 0.8946 & 0.5000 & 0.5484 & 0.8750 & $4.39(1.15-16.68)$ \\
\hline Hysterectomy & 0.8333 & 0.5185 & 0.5357 & 0.8235 & $3.04(1.03-8.97)$ \\
\hline
\end{tabular}

PPV, positive predictive value; NPV, negative predictive value; RR, relative risk; CI, confidence interval. 
different from the cases treated with hysterectomy $(P>0.05$, Fisher's exact test).

To determine whether podoplanin expression correlates with patient outcome, first we analyzed all cases combined (Series A and B, total 122 cases). During the follow-up interval, tumor progression or recurrence was observed in 48 (39\%) cases. Twentynine $(24 \%)$ patients died of disease at a median time of 18.9 months (range 4.4-95.0 months) of followup. The median follow-up of censored patients was 44.0 months (range 0-188.1 months). The median time to tumor recurrence was 11.9 months (range 069.3 months). In the combined series, low podoplanin expression was highly significantly associated with both poor overall $(P=0.0008)$ and disease-free $(P=0.0014)$ survival (Figure $3 \mathrm{a}$ and $\mathrm{b})$.

Treatment options for patients with stage IB and IIA cervix cancers include either radical surgery or primary radiation therapy. Thus, we analyzed this group of patients separately. In the combined series, there were 60 patients (48 stage IB and 12 stage IIA) in this group. Primary treatment was radical hysterectomy in 38 cases, while 22 patients received primary radiation. During the follow-up interval, tumor recurrence was observed in $20(33 \%)$ cases. A total of $11(18 \%)$ patients died of disease at a median time of 24.1 months (range 10.6-95.0 months) of follow-up. The median follow-up of censored patients was 56.3 months (range 0-181.3 months). The median time to tumor recurrence was 15.3 months (range 0-69.3 months). In this subgroup of patients, the method of primary treatment showed no correlation with either overall or disease-free survival (Figure 3c and d). In contrast, low podoplanin expression in pretreatment biopsy samples was significantly associated with both poor overall $(P=0.0251)$ and disease-free $(P=0.0159)$ survival (Figure $3 e$ and $\mathrm{f}$ ).

Among the 74 patients treated with primary radiation, tumor progression or recurrence was observed in $36(49 \%)$ cases during the follow-up interval. A total of $25(34 \%)$ patients died of disease at a median time of 18.5 months (range 4.4-95.0 months) of follow-up. The median follow-up of censored patients was 55.6 months (range 0.9-188.1 months). The median time to tumor recurrence was 10.5 months (range 0-60.2 months). We found no correlation between tumor size, grade, FIGO stage, or total radiation dose and either disease-free or overall survival, although FIGO stage (IB vs IIB vs stage III and IV) approached statistical significance for overall survival $(P=0.0524)$. In this group of patients treated by radiation, low podoplanin expression in the pretreatment biopsy material was highly significantly associated with both poor overall $(P=0.0002)$ and disease-free $(P=0.0009)$ survival (Figure $3 \mathrm{~g}$ and $\mathrm{h}$ ). For stepwise logistic regression, we included in the models FIGO stage, tumor grade, tumor size, total radiation dose, and podoplanin expression. Backward elimination for Cox regression led to a model with one independent term predictive of overall and recurrence-free survival: podoplanin expression $(P=0.006$ and $P=0.022$, respectively). The sensitivity, specificity, and positive and negative predictive values of low podoplanin expression to predict the presence of tumor progression/recurrence and poor overall outcome (dying from disease) in cervical squamous cell carcinomas are summarized in Table 3.

\section{Discussion}

The recently developed monoclonal antibody D2-40 detects a fixation-resistant epitope on podoplanin, ${ }^{12,13}$ a mucin-type transmembrane sialoglycoprotein specifically expressed on lymphatic endothelial cells, fetal germ cells, and kidney podocytes. ${ }^{20-22}$ Antibodies to podoplanin, including D2-40, were reported to be selective markers for lymphatic endothelium and to be useful in identifying the presence of lymphatic invasion in various malignant neoplasms, including cervical carcinoma. ${ }^{15-17}$ However, little is known about the biological function of podoplanin. It was recently reported that mice deficient in podoplanin develop congenital lymphedema and have defects in lymphatic vessel, but not blood vessel, formation. ${ }^{23}$ In vitro studies suggested that podoplanin may be involved in mediating cell motility and adhesion. ${ }^{23,24}$ Very recently, podoplanin expression was reported in squamous cell carcinomas of the epidermis, ${ }^{13}$ oral cavity, ${ }^{24}$ and lung, ${ }^{25}$ and it was suggested that it may be involved in tumor cell invasion, metastasis, and tumor progression. In addition, mucin-type glycoproteins, such as podoplanin, are expressed widely on human normal cells and tumors ${ }^{26-28}$ and have recently been implicated in cellular signaling, cell adhesion, tumor growth, and suppression of apoptosis. ${ }^{29-33}$

We have recently characterized the immunohistochemical reactivity of tumor cells in a series of earlystage cervical squamous cell carcinomas with the D2-40 antibody and found that low levels of podoplanin expression in the tumor cells were significantly associated with the presence of lymphatic invasion and lymph node metastasis, as well as with shorter survival and higher risk of tumor recurrence. ${ }^{16}$ Interestingly, we found that tumor emboli within lymphatic spaces, as well as metastatic tumor cells in lymph nodes, showed no podoplanin immunostaining in the vast majority of tumors, even in cases when the main tumor mass was D2-40 positive. While the importance of the lymphatic system as a pathway for metastasis has been well recognized, there is very little information available about the mechanisms by which tumor cells interact with the lymphatics. Our results suggested that podoplanin may play a role in the interaction of tumor cells with the lymphatics.

The presence of lymphovascular invasion is a significant risk factor for tumor recurrence in cervical squamous cell carcinoma. ${ }^{4-6}$ Many patients 

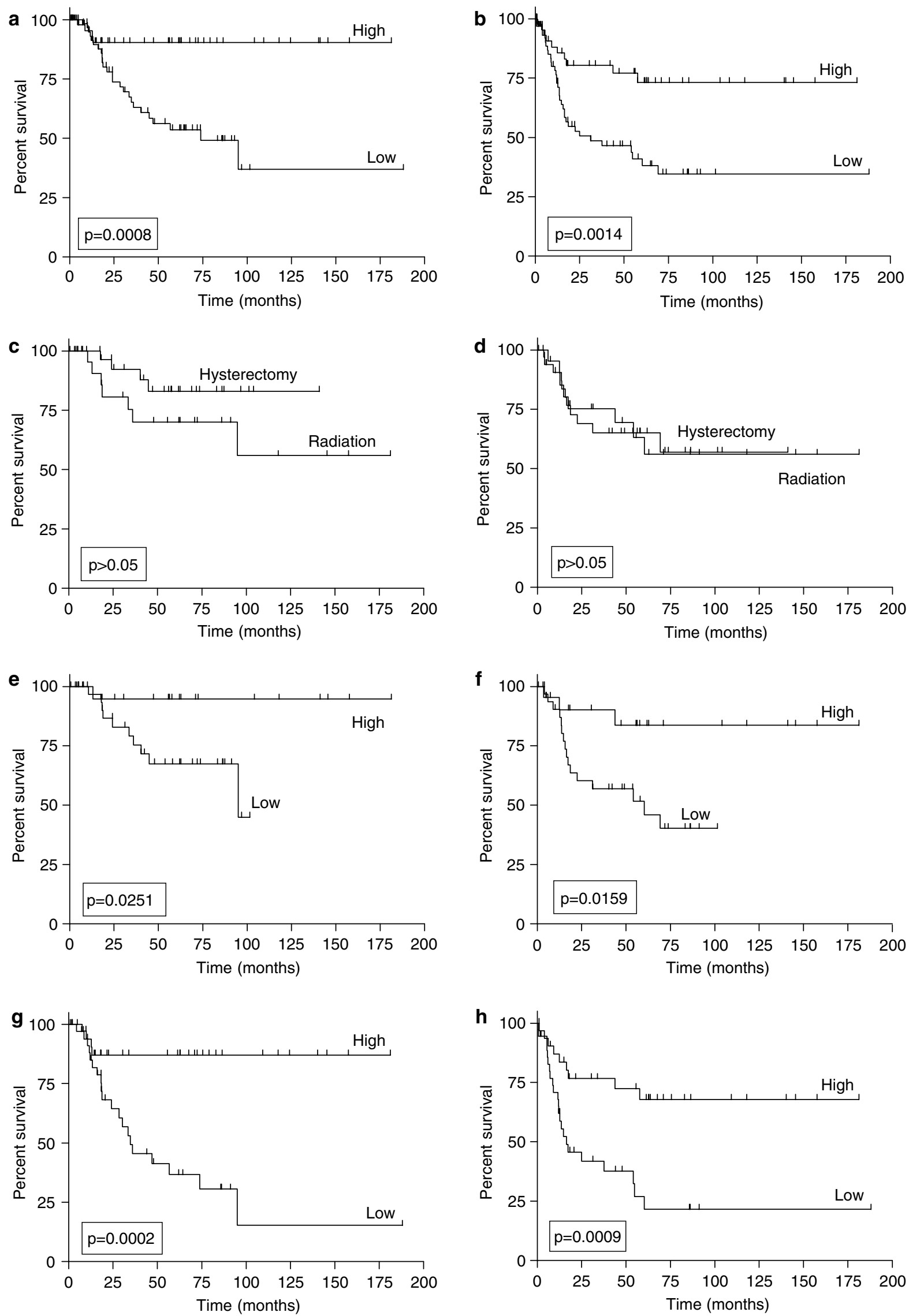
Table 3 Sensitivity, specificity, positive and negative predictive value of low podoplanin expression to predict tumor progression/ recurrence and poor overall outcome (dying from disease) in cervical squamous cell carcinomas

\begin{tabular}{|c|c|c|c|c|c|}
\hline & Sensitivity & Specificity & $P P V$ & $N P V$ & $R R(95 \% C I)$ \\
\hline \multicolumn{6}{|c|}{ All cases (series $A$ and $B$ ) combined } \\
\hline Recurrence & 0.5294 & 0.7692 & 0.7500 & 0.5556 & $1.69(1.24-2.30)$ \\
\hline Dying from disease & 0.3676 & 0.9231 & 0.8621 & 0.5275 & $1.82(1.41-2.37)$ \\
\hline \multicolumn{6}{|c|}{ Advanced stage cases treated by primary radiation } \\
\hline Recurrence & 0.6579 & 0.6765 & 0.6944 & 0.6389 & $1.92(1.18-3.13)$ \\
\hline Dying from disease & 0.5526 & 0.8824 & 0.8400 & 0.6383 & $2.32(1.53-3.52)$ \\
\hline
\end{tabular}

PPV, positive predictive value; NPV, negative predictive value; RR, relative risk; CI, confidence interval.

with advanced-stage disease are treated primarily with nonsurgical methods after biopsy confirmation of the diagnosis. However, the presence of lymphatic invasion might be underestimated in biopsy material due to the size of tumor sampling. Currently, there are no reliable histopathologic features or biological tumor markers to predict the presence of lymphatic invasion and nodal metastasis, or to provide prognostic information in advanced-stage tumors based on such small biopsy materials. In the present study, we have shown that evaluation of podoplanin expression by D2-40 immunohistochemistry in small cervical biopsy specimens is equally effective as hysterectomy materials to predict the presence of lymphatic invasion and/or lymph node metastasis in cervical cancer. This finding could be significant for patients with advanced-stage disease treated by nonsurgical means and in which conventional histologic prognostic factors might be underestimated in small biopsy samples.

In addition, as in the series of early-stage cervical cancers, ${ }^{16}$ we have also shown that low podoplanin expression in the tumors based on pretreatment biopsy materials is highly significantly associated with an increased risk of tumor recurrence and shorter overall survival in cervix cancer patients irrespective of the mode of primary therapy in both univariate and multivariate analysis.

Our findings demonstrate that podoplanin expression determined by the D2-40 immunostaining pattern in biopsy material reliably reflects that of the main tumor in hysterectomy specimens, and can predict the status of lymphatic involvement as well as clinical outcome. While the sensitivity and specificity of determination of podoplanin immunoreactivity to predict lymphatic invasion and nodal metastasis appears to be high enough to be of potential clinical utility in the workup of cervix cancer patients, further studies are needed to establish its clinical utility. Although prediction of para-aortic lymph node involvement specifically would be important to aid in clinical decisionmaking and treatment planning, only five patients included in the current study had para-aortic node involvement, precluding separate analysis. Future studies are needed to examine whether podoplanin expression would be useful in predicting para-aortic lymph node status.

In summary, low podoplanin expression in small biopsy or hysterectomy specimens is highly significantly associated with lymphatic space involvement and nodal metastasis as well as an increased risk of recurrence and poor outcome in advanced-stage cervical squamous cancers. Assessment of podoplanin expression by D2-40 immunostaining performed on cervical biopsy material appears to be a valuable test to predict lymph node status in patients with unresectable disease undergoing primary nonsurgical treatment, when biopsy tumor tissue is less than optimal for thorough evaluation of conventional prognostic factors, as well as to provide additional prognostic information in advanced-stage cervical cancer. Prospective studies involving larger numbers of patients are needed to further evaluate the clinical utility of examination of podoplanin expression in patients with cervix carcinoma.

\section{References}

1 Sleeman JP. The lymph node as a bridgehead in the metastatic dissemination of tumors. Recent results. Cancer Res 2000;157:55-81.

2 Stacker SA, Achen MG, Jussila L, et al. Lymphangiogenesis and cancer metastasis. Nat Rev Cancer 2002; 2:573-583.

3 Stacker SA, Baldwin ME, Achen MG. The role of tumor lymphangiogenesis in metastatic spread. FASEB J 2002;16:922-934.

4 Van Bommel PF, van Lindert AC, Kock HC, et al. A review of prognostic factors in early-stage carcinoma of the cervix (FIGO I B and II A) and implications for treatment strategy. Eur J Obstet Gynecol Reprod Biol 1987;26:69-84.

Figure 3 Kaplan-Meier overall (a, c, e, g) and recurrence-free (b, d, f, h) survival curves in all cases (a, b), stage IB and IIA cases (c-f), and cases treated by primary radiation therapy $(\mathbf{g}, \mathbf{h})$. Curves are stratified for low $v s$ high podoplanin expression $(\mathbf{a}, \mathbf{b}, \mathbf{e}-\mathbf{h})$, or primary mode of treatment $(\mathbf{c}, \mathbf{d})$. 
5 Hoskins WJ. Prognostic factors for risk of recurrence in stages Ib and IIa cervical cancer. Baillieres Clin Obstet Gynaecol 1988;2:817-828.

6 Robert ME, Fu YS. Squamous cell carcinoma of the uterine cervix - a review with emphasis on prognostic factors and unusual variants. Semin Diagn Pathol 1990;7:173-189.

7 Lai CH, Huang KG, Hong JH, et al. Randomized trial of surgical staging (extraperitoneal or laparoscopic) vs clinical staging in locally advanced cervical cancer. Gynecol Oncol 2003;89:160-167.

8 Piver MS, Barlow JJ, Krishnamsetty R. Five-year survival (with no evidence of disease) in patients with biopsy-confirmed aortic node metastasis from cervical carcinoma. Am J Obstet Gynecol 1981;139: $575-578$.

9 Twiggs LB, Potish RA. Decision theory analysis of the enteric morbidity and surgical staging in the treatment of advanced cervical cancer. Am J Obstet Gynecol 1984;148:134-140.

10 Haie C, Pejovic MH, Gerbaulet A, et al. Is prophylactic para-aortic irradiation worthwhile in the treatment of advanced cervical carcinoma? Results of a controlled clinical trial of the EORTC radiotherapy group. Radiother Oncol 1988;11:101-112.

11 Rotman M, Pajak TF, Choi K, et al. Prophylactic extended-field irradiation of para-aortic lymph nodes in stages IIB and bulky IB and IIA cervical carcinomas. Ten-year treatment results of RTOG 79-20. JAMA 1995;274:387-393.

12 Marks A, Sutherland DR, Bailey D, et al. Characterization and distribution of an oncofetal antigen (M2A antigen) expressed on testicular germ cell tumours. Br J Cancer 1999;80:569-578.

13 Schacht V, Dadras SS, Johnson LA, et al. Up-regulation of the lymphatic marker podoplanin, a mucin-type transmembrane glycoprotein, in human squamous cell carcinomas and germ cell tumors. Am J Pathol 2005;166:913-921.

14 Franchi A, Gallo O, Massi D, et al. Tumor lymphangiogenesis in head and neck squamous cell carcinoma: a morphometric study with clinical correlations. Cancer 2004;101:973-978.

15 Kahn HJ, Marks A. A new monoclonal antibody, D2-40, for detection of lymphatic invasion in primary tumors. Lab Invest 2002;82:1255-1257.

16 Dumoff KL, Chu C, Xu X, et al. Low D2-40 immunoreactivity correlates with lymphatic invasion and nodal metastasis in early-stage squamous cell carcinoma of the uterine cervix. Mod Pathol 2005;18: 97-104.

17 Gombos Z, Xu X, Chu CS, et al. Peritumoral lymphatic vessel density and vascular endothelial growth factor$\mathrm{C}$ expression in early-stage squamous cell carcinoma of the uterine cervix. Clin Cancer Res 2005;11: 8364-8371.

18 Pecorelli S, Benedet JL, Creasman WT, et al. FIGO staging of gynecologic cancer. 1994-1997 FIGO Committee on Gynecologic Oncology. International
Federation of Gynecology and Obstetrics. Int J Gynaecol Obstet 1999;65:243-249.

19 Kurman RJ, Norris HJ, Wilkinson EJ. Tumors of the Cervix, Vagina, and Vulva. Armed Forces Institute of Pathology: Washington, DC, 1992.

20 Hirakawa S, Hong YK, Harvey N, et al. Identification of vascular lineage-specific genes by transcriptional profiling of isolated blood vascular and lymphatic endothelial cells. Am J Pathol 2003;162:575-586.

21 Wetterwald A, Hoffstetter W, Cecchini MG, et al. Characterization and cloning of the E11 antigen, a marker expressed by rat osteoblasts and osteocytes. Bone 1996;18:125-132.

22 Breiteneder-Geleff S, Matsui K, Soleiman A, et al. Podoplanin, novel 43-kD membrane protein of glomerular epithelial cells, is down-regulated in puromycin nephrosis. Am J Pathol 1997;151:1141-1152.

23 Schacht V, Ramirez MI, Hong YK, et al. T1alpha/ podoplanin deficiency disrupts normal lymphatic vasculature formation and causes lymphedema. EMBO J 2003;22:3546-3556.

24 Martin-Villar E, Scholl FG, Gamallo C, et al. Characterization of human PA2.26 antigen (T1alpha-2, podoplanin), a small membrane mucin induced in oral squamous cell carcinomas. Int J Cancer 2005;113: 899-910.

25 Kato Y, Kaneko M, Sata M, et al. Enhanced expression of Aggrus (T1alpha/podoplanin), a platelet-aggregation-inducing factor in lung squamous cell carcinoma. Tumour Biol 2005;26:195-200.

26 Sutherland DR, Rudd CE, Greaves MF. Isolation and characterization of a human T lymphocyte-associated glycoprotein (gp40). J Immunol 1984;133:327-333.

27 Carraway KL, Hull SR. O-glycosylation pathway for mucin-type glycoproteins. BioEssays 1989;10:117-121.

28 Lesuffleur T, Zweibaum A, Real FX. Mucins in normal and neoplastic human gastrointestinal tissues. Crit Rev Oncol Hematol 1994;17:153-180.

29 Klapper LN, Kirschbaum MH, Sela M, et al. Biochemical and clinical implications of the ErbB/HER signaling network of growth factor receptors. Adv Cancer Res 2000;77:25-79.

30 Carraway KL, Perez A, Idris N, et al. Muc4/sialomucin complex, the intramembrane ErbB2 ligand, in cancer and epithelia: to protect and to survive. Prog Nucleic Acid Res Mol Biol 2002;71:149-185.

31 Komatsu M, Jepson S, Arango ME, et al. Muc4/ sialomucin complex, an intramembrane modulator of ErbB2/HER2/Neu, potentiates primary tumor growth and suppresses apoptosis in a xenotransplanted tumor. Oncogene 2001;20:461-470.

32 Yamamoto M, Bharti A, Li Y, et al. Interaction of the DF3/MUC1 breast carcinoma-associated antigen and beta-catenin in cell adhesion. J Biol Chem 1997;272: 12492-12494.

33 Wesseling J, van der Valk SW, Vos HL, et al. Episialin (MUC1) overexpression inhibits integrin-mediated cell adhesion to extracellular matrix components. J Cell Biol 1995;129:255-265. 